\title{
Octadecadienoic Acid n-9
}

National Cancer Institute

\section{Source}

National Cancer Institute. Octadecadienoic Acid n-9. NCI Thesaurus. Code C68402.

A polyunsaturated long-chain fatty acid with an 18-carbon backbone and double bonds originating from the 9 th position and the 11th, 12th, 13th or 14th positions from the methyl end. There are at least 7 different isomers that can be called by this name. 